\title{
纤维状多孔钙锰氧化物制备及其氧还原反应电催化活性
}

\author{
曹朝霞，丁延敏，王志超，毛新欣，尹艳红，杨书廷 \\ (1. 河南师范大学 化学化工学院, 新乡 453007; 2. 河南省电池研究院 新乡 453000)
}

摘 要: 首次以海藻酸钲纤维为载体制得层状-钻钠矿结构的 $\mathrm{Ca}_{2} \mathrm{MnO}_{4}$ 䥻锰氧化物(Ca-Mn-O), 并以尖晶石结构的 $\mathrm{CaMn}_{3} \mathrm{O}_{6}$ 为对比样, 测试了样品作为电化学氧还原催化剂(ORR)的性能。通过 XRD、FE-SEM、TEM 和 BET 对材 料进行表征，结果表明该层状-钙钠矿结构的氧化物具有内部相互交联的多孔网状结构和大的比表面积。电催化测 试结果表明: $\mathrm{Ca}_{2} \mathrm{MnO}_{4}$ 氧化物具有明显的催化活性, 高的极限扩散电流密度、接近四电子氧化还原以及在碱性溶液 中较低产率的过氧化氢, 说明这种廉价而丰富的 ORR 催化剂具有潜在的应用前景。Ca-Mn-O 氧化物的催化活性(初 始还原电位、极限电流密度以及电子转移数)很大程度依赖于表面 $\mathrm{Mn}$ 的氧化态和晶体结构。此外, 大的比表面积 和多孔网状结构, 增多了氧还原反应的电催化活性位; 高的氧空位浓度有利于氧还原反应的进行; 合适的晶体结 构具有开放的空间，有利于氧的吸附。

关 键 词: 海藻酸钲纤维; 非贵金属催化剂; $\mathrm{Ca}_{2} \mathrm{MnO}_{4}$ 氧还原; 燃料电池

中图分类号: TM912 文献标识码: A

\section{Porous Calcium Manganese Oxide: Preparation and Electrocatalytic Activity of Oxygen Reduction Reaction}

\author{
CAO Zhao-Xia, DING Yan-Min, WANG Zhi-Chao, MAO Xin-Xin, YIN Yan-Hong, YANG Shu-Ting \\ (1. College of Chemistry and Chemical Engineering, Henan Normal University, Xinxiang 453007, China; 2. Henan Battery \\ Research Institute, Xinxiang 453000, China)
}

\begin{abstract}
Calcium-manganese oxides (Ca-Mn-O) of layer-pervoskite $\mathrm{Ca}_{2} \mathrm{MnO}_{4}$ was firstly prepared by using calcium alginate fiber as supporter and then investigated as electrocatalysts for oxygen reduction reaction (ORR), compared with post-spinel $\mathrm{CaMn}_{3} \mathrm{O}_{6}$. The results of XRD, FE-SEM, TEM and BET displayed the as-synthesized layer-pervoskite $\mathrm{Ca}_{2} \mathrm{MnO}_{4}$ displayed interconnected porous network structure and high special surface area. The catalyst demonstrates significant electrocatalytic activity towards ORR: a high limit current density, near an apparent quasi four electron oxygen reduction, and low yield of peroxide species in alkaline solutions, which suggestes the potential application of $\mathrm{Ca}_{2} \mathrm{MnO}_{4}$ as cheap and abundant ORR catalysts. The catalytic activities of Ca-Mn-O oxides (onset potential, limiting current density and transferred electron number) depend strongly on the surface Mn oxidation state and the crystallographic structures. In addition, high specific surface area and porous network structure increase electrocatalytic activity sites of ORR, high concentration of oxygen vacancies favor process of ORR, appropriate crystallographic structures with open tunnels, facilitating oxygen chemisorption.
\end{abstract}

收稿日期: 2016-07-22; 收到修改稿日期：2016-09-30

基金项目: 河南省科技攻关项目(152102210080); 河南省教育厅科学技术研究重点项目(12B480002); 河南省科技攻关计划 (国际科技合作)(162102410047)

Scientific and Technologies Research Program of Henan Province (152102210080); Key Research Program of Scientific and Technologies of Henan Provincial Department of Education (12B480002); Science and technology project of Henan Province (International Cooperation in Science and Technology) (162102410047)

作者简介：曹朝霞(1979-), 女，博士，副教授. E-mail: zxcao@foxmail.com

通讯作者: 杨书廷，教授. E-mail: shutingyang@foxmail.com 
Key words: calcium alginate fibre; non-noble metal catalysts; $\mathrm{Ca}_{2} \mathrm{MnO}_{4}$ oxygen reduction; fuel cell

随着传统化石燃料的过渡消耗和对生活环境的 严重污染, 人们对清洁能源的需求不断提高。燃料 电池以其高功率密度、高能量转换效率、室温快速 启动和零污染等优点, 成为最具有前途的绿色能源 之一 ${ }^{[1-3]}$ 。目前, 燃料电池主要以价格高昂、资源稀 少的铂及其合金化合物等作为氧还原反应 $(\mathrm{ORR})$ 的 催化剂, 这严重制约了在实际中的应用 ${ }^{[4-6]}$ 。因此, 需要寻找廉价的高催化活性的非铂催化剂。

近些年, 各种过渡金属氧化物已在电化学氧催 化方面得到广泛研究, 发现氧化物自身的 ORR 活 性与 $\sigma$-反键轨道的占用以及表面过渡金属原子共价 状态密切相关 ${ }^{[7]}$ 。锰基氧化物具有价格低廉、储量 丰富和可变价态 $\left(\mathrm{Mn}^{2+} 、 \mathrm{Mn}^{3+} 、 \mathrm{Mn}^{4+} 、 \mathrm{Mn}^{6+}\right.$ 和 $\left.\mathrm{Mn}^{7+}\right)$ 等特点, 可以形成多种结构的氧化物及复合氧化物 (钙钛矿, 尖晶石类等), 并且展示出较好的 ORR 活 性而成为研究热点 ${ }^{[8-13]}$ 。对于锰氧化物来说, 大多数 催化剂中 $\mathrm{Mn}$ 的平均价态在 $\mathrm{Mn}(\mathrm{III})-\mathrm{Mn}(\mathrm{IV})$, 该类混 合价态的锰氧化物提供了催化剂表面与反应物或生 成物之间的强结合能力。不同于贵金属催化剂, 过 渡金属氧化物的 ORR 催化是通过表面氧化还原伴 随着电子转移至氧, 锰氧化物的多价和高价态有利 于氧的活化, 得到更好地 ORR 动力学 ${ }^{[14]}$ 。有报道发 现高价态的 $\mathrm{Mn}$ 具有较高的 ORR 活性, 原位 XANES 结果也表明, Mn (IV)含量高的 $\mathrm{MnO}_{x}$ 材料展 现出更好地催化性能 ${ }^{[15]}$ 。

近期研究表明, 将钙掺入锰氧化物可以较大程 度地改善它们的水氧化活性和作为钠空气电池正极 材料的性能 ${ }^{[16]}$ 。应该注意的是, $\mathrm{Ca}$ 对 $\mathrm{Ca}-\mathrm{Mn}-\mathrm{O}$ 氧化 物的 ORR 电催化过程也有重要的作用, 可以影响 表面氧的吸附、活化和还原。由于氧化物晶格中氧 的协调性, 钙离子与高价锰的结合提高了晶体的化 学和结构稳定性。Han 等 ${ }^{[17]}$ 使用固相烧结法制备了 不同晶型的氧化物, 同时报道了钙钛矿结构的 $\mathrm{CaMnO}_{3}$ 、层状结构的 $\mathrm{Ca}_{2} \mathrm{Mn}_{3} \mathrm{O}_{8}$ 和后尖晶石型 $\mathrm{CaMn}_{2} \mathrm{O}_{4} 、 \mathrm{CaMn}_{3} \mathrm{O}_{6}$ 作为 ORR 反应的催化剂具有较 好的活性, 但层状一钙钛矿结构的 $\mathrm{Ca}_{2} \mathrm{MnO}_{4}$ 氧化物 作为 ORR 反应催化剂的相关研究却尚未见报道。

本工作采用海藻酸钙纤维为载体, 利用离子交 换法使锰离子置换部分钲离子, 采用水热法原位制 备钙锰纤维前驱体, 经过炦烧得到内部交联的多孔 状 $\mathrm{Ca}_{2} \mathrm{MnO}_{4}$ 氧化物, 并以 $\mathrm{CaMn}_{3} \mathrm{O}_{6}$ 作为对比样, 研 究了该材料的物理性能, 利用旋转环盘电极对其 ORR 催化性能进行评估。

\section{1 实验方法}

\subsection{Ca-Mn-O 氧化物的制备}

(1) 称取 $6.7 \mathrm{~g}$ 的醋酸锰加入 $100 \mathrm{~mL}$ 的蒸馏水 中, 搅拌至溶解, 然后加入 $1.5 \mathrm{~g}$ 的海藻酸钻纤维, 继续摚拌 $24 \mathrm{~h}$ 。超声 $30 \mathrm{~min}$ 之后, 对其进行抽滤, 用 $500 \mathrm{~mL}$ 的蒸馏水洗涤。经过多次抽滤、洗涤后, 将 纤维放进 $80^{\circ} \mathrm{C}$ 的鼓风干燥箱中进行干燥, 待用。

(2) 分别称取 $0.052 \mathrm{~g}$ 和 $1.488 \mathrm{~g}$ 醋酸锰, 溶解在 含有 $50 \mathrm{~mL}$ 蒸馏水的烧杯中, 随后将 $0.5 \mathrm{~g}$ 处理过的 海藻酸钻纤维加入上述溶液中, 摚拌 $10 \mathrm{~min}$ 后, 将 上述混合物转移至 $100 \mathrm{~mL}$ 的反应釜，设置反应温 度为 $120^{\circ} \mathrm{C}$, 保温时间为 $6 \mathrm{~h}$ 。反应结束待自然冷却 后, 对样品进行抽滤, 并用定量的蒸馏水洗涤, 在 $80^{\circ} \mathrm{C}$ 条件下烘干。

(3) 将水热反应产物在 $750^{\circ} \mathrm{C}$ 、空气气氛下、保 温 $2 \mathrm{~h}$ 进行㷽烧, 最后得到 Ca-Mn-O 产物, 根据醋 酸锰用量多少 (0.052 g 和 $1.488 \mathrm{~g})$, 分别命名为 CMO-1 和 $\mathrm{CMO}-2$ 。

\subsection{Ca-Mn-O 氧化物的表征}

使用 D8 型 X 射线衍射仪(德国布鲁克 $\mathrm{AXS}$ 公司) 分析样品的晶体结构, 靶源为 $\mathrm{Cu} \mathrm{Ka}$ 射线 $(\lambda=$ $0.1504 \mathrm{~nm}$ ), 工作电流及电压分别为 $20 \mathrm{~mA}$ 和 $40 \mathrm{kV}$, 扫描范围为 $10^{\circ} \sim 80^{\circ}$ 。采用场发射扫描电镜(FE-SEM; SU8010)、能量色散谱仪(EDS)和日本 JEOL JEM2100 型电子显微镜进行形貌观察、元素分布分析和 微观结构观察。由美国 Thermo 生产的 ESCALAB250 型 X 射线光电子能谱仪分析表面元 素组成, 以 $\mathrm{C} 1 \mathrm{~s}(284.6 \mathrm{eV})$ 为参考线。采用物理吸附 分析仪 Tristar II 3020 对所制备的样品进行比表面 积和孔径分布分析。

\section{3 电催化性能测试}

电极制备: 称取 $3 \mathrm{mg}$ 催化剂和 $7 \mathrm{mg}$ 乙炔黑, 研磨均匀。加入 $700 \mu \mathrm{L}$ 异丙醇和 $190 \mu \mathrm{L}$ Nafion 溶 液, 将混合物超声分散 $30 \mathrm{~min}$, 得到均匀分散的悬 浊液。移取 $8.75 \mu \mathrm{L}$ 催化剂悬浊液涂在盘电极的表 面, 空气中干燥后作为工作电极。

循环伏安扫描 $(\mathrm{CV})$ 分析法: 采用常规的三电极 电解池进行电化学性能测试, 其中以铂丝为对电极, $\mathrm{Ag} / \mathrm{AgCl}$ 为参比电极, $0.1 \mathrm{~mol} / \mathrm{L} \mathrm{KOH}$ 为电解液, 向 电解池中通氮气(氧气)至饱和, 并在实验过程中持 续通氮气(氧气) 以保持溶液上方的气氛。利用恒电 
流电化学工作站和旋转环盘电极进行测量。参比电 极 $\mathrm{Ag} / \mathrm{AgCl}$ 与可逆氢电极 (RHE) 之间的电位按 $E$ $(\mathrm{RHE})=E(\mathrm{Ag} / \mathrm{AgCl})+0.982 \mathrm{~V}\left(0.1 \mathrm{~mol} / \mathrm{L} \mathrm{KOH}, 25^{\circ} \mathrm{C}\right)$ 进行换算 ${ }^{[18]}$ 。

碱性体系中的催化氧还原测试: 通常采用旋转 环盘电极技术测试催化 ORR 活性, 测试过程中, 保 持氧气进气管在溶液液面以下电极水平面以上的位 置鼓气, 从开路电势向负电势方向循环伏安扫描, 测试温度为 $25^{\circ} \mathrm{C}$ 。

\section{2 结果与讨论}

\section{1 结构与形貌}

\subsubsection{XRD、比表面积及孔径分布表征}

图 1(a)是 Ca-Mn-O 氧化物材料的 XRD 图谱。 对比标准卡片之后, 发现样品 CMO-1 与 $\mathrm{Ca}_{2} \mathrm{MnO}_{4}$ (JCPDS 24-1194)的标准 XRD 图谱相匹配。对比样 CMO-2 的 XRD 图谱与标准 $\mathrm{CaMn}_{3} \mathrm{O}_{6}$ (JCPDS 31-0285) 的衍射峰相匹配。并且, 两种材料都具有较强的衍 射峰，表明它们都有良好的结晶性。

高温敾烧可以使海藻酸纤维发生热裂解, 产物 脱酸释放 $\mathrm{CO}_{2}$ 和 $\mathrm{CO}$, 有利于形成多孔的网状结构, 从而提高氧化物的比表面积。图 1(b)给出了样品的 等温吸脱附曲线, 从图中可以看出, 该类吸脱附曲 线属于IV类型，内插图是由 BJH 方法计算出的孔径 分布曲线, 这两条曲线具有相似的特征, 从中可以 看出一部分小孔的孔径位于 $30 \sim 40 \mathrm{~nm}$, 而大部分孔 径主要集中在 100 110 nm。样品 CMO-1 具有更大 的比表面积和孔体积 (21.6 vs $8.15 \mathrm{~m}^{2} / \mathrm{g}, 0.179 \mathrm{~m}^{3} / \mathrm{g}$ vs $0.051 \mathrm{~m}^{3} / \mathrm{g}$ )。这种方法制得的 Ca-Mn-O 氧化物多 孔结构大大增加了催化剂的比表面积, 进而可以增 强它们对氧还原反应的电催化活性。

\subsubsection{FE-SEM 和 EDS 表征}

图 2 是海藻酸钙纤维的扫描电镜照片, 从图中

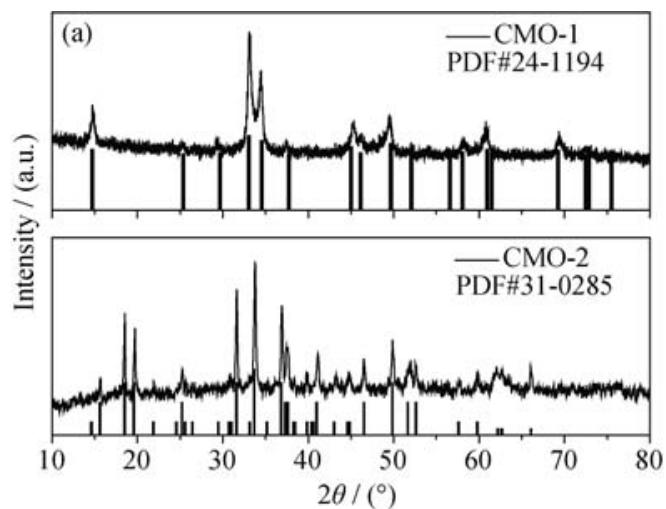

可以看出纤维的表面光滑。经过炦烧, 各样品保持 了纤维原有的形态, 如图 3(a)(CMO-1) 和 3c (CMO-2) 所示。图 3(b)表明 CMO-1 的纤维状截面出现一些片 状、相互交联的多孔状结构, 并含有较多的大孔; 内 插图中可以看到表面有均匀的颗粒状，直径为 20 40 nm, 且伴随着大量的小孔，这种多孔结构增 多了反应位点, 有利于氧气的扩散和电解液的浸润, 从而提高了反应速率。从图 3(d)中明显看出, CMO-2 的表面颗粒物变大、团聚现象严重，这是由于锰盐 浓度增加以及奥斯特瓦尔德熟化机理使其发生了团 聚现象 ${ }^{[19]}$ 。选取 CMO-1 样品做选区元素分析, 如图 3(e)所示, 可以看出样品是由 $\mathrm{Mn} 、 \mathrm{Ca}$ 和 $\mathrm{O}$ 三种元 素组成, 图 3f 中则分别以绿色、黄色、红色表示了 $\mathrm{Mn} 、 \mathrm{Ca}$ 和 $\mathrm{O}$ 元素, 从中能清晰地看到这些元素分 布均匀。

\subsubsection{TEM 表征}

图 4 是 Ca-Mn-O 氧化物的 TEM 和 HRTEM 照 片。从图 4(a)和 4(d)可以看出两种材料均呈内部相 互交联的多孔网状结构, 从对应的放大图 4(b)可以 发现, CMO-1 颗粒约为 30 nm, 颗粒间还有堆积的 孔。晶格条纹间距 $0.62 \mathrm{~nm}$ 和 $0.28 \mathrm{~nm}$ 分别对应于 $\mathrm{Ca}_{2} \mathrm{MnO}_{4}$ 的(002) 和 (103) 晶面(图 4(c))。CMO-2 的晶粒明显增大，约在 $50 \sim 100 \mathrm{~nm}$, 并伴随有分布 不均匀的现象(图 4(e)), 其晶格条纹间距 $0.27 \mathrm{~nm}$ 和 $0.57 \mathrm{~nm}$ 分别对应于 $\mathrm{CaMn}_{3} \mathrm{O}_{6}$ 的特征晶面(图 4(f))。 这与 XRD 测试结果是一致的。

\subsubsection{XPS 表征}

过渡金属氧化物的 ORR 电催化反应机理，包括 催化剂表面的氧气吸附、解离和还原，这与过渡金 属的氧化还原反应是密切相关的。因此，合理的化 学成分、元素价态、晶体结构和表面状态是决定催 化活性的重要因素。实验通过 X 射线光电子能谱 (XPS)对氧化物的表面元素价态进行了分析，结果 如图 5 所示, 两者的全谱图中都有 $\mathrm{Mn}(2 \mathrm{~s}, 2 \mathrm{p}, 3 \mathrm{~s}$ 和

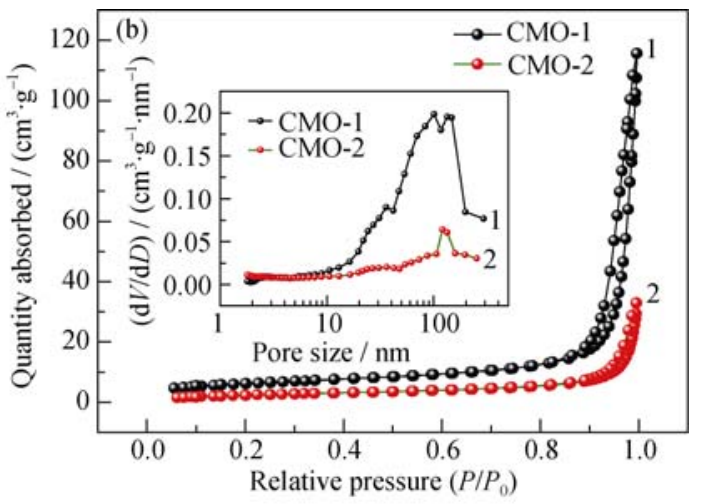

图 $1 \mathrm{Ca}-\mathrm{Mn}-\mathrm{O}$ 材料的 XRD 图谱(a), 等温吸脱附曲线和孔径分布图(b)

Fig. 1 XRD patterns (a), adsorption-desorption isothermal and inset of pore size distribution curves (b) of Ca-Mn-O samples 


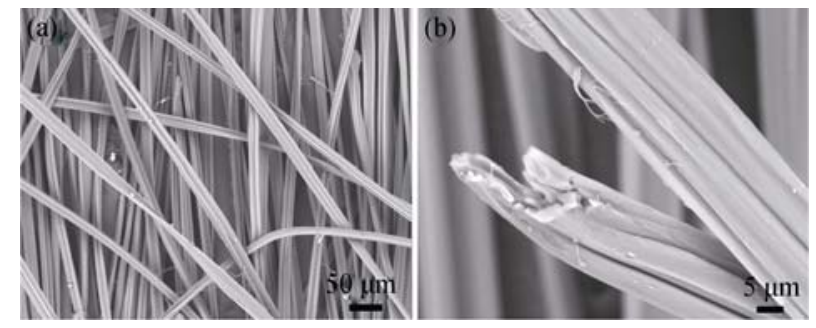

图 2 海藻酸钲纤维的 SEM 照片

Fig. 2 SEM images of calcium alginate fibre

$3 p), \mathrm{O}(1 \mathrm{~s}, \mathrm{KLL})$ 和 $\mathrm{Ca}(2 \mathrm{~s}, 2 \mathrm{p})$ 的特征峰, 说明材料都 由 $\mathrm{Mn} 、 \mathrm{O}$ 和 $\mathrm{Ca}$ 三种元素组成。

$\mathrm{O} 1 \mathrm{~s}$ 的光电子能谱在约 529 和 $531 \mathrm{eV}$ 分别对应 于 $\mathrm{M}-\mathrm{O}$ (晶格氧)和 $\mathrm{O}-\mathrm{O}$ 键(表面吸附氧)。表面吸附 氧有利于 ORR 催化性能, 这与氧空位紧密联系。氧 空位随着 $\mathrm{Ca}$ 含量的增加而增多, 所以吸附氧含量也 随之增加。从图 5 还可以明显看出 $\mathrm{Ca}_{2} \mathrm{MnO}_{4}$ 中表面 吸附氧与晶格氧的峰值强度比相对较大, 较多的表 面吸附氧有利于 ORR 催化活性 ${ }^{[20-21]}$ 。根据文献[17] 报道, 由于 $\mathrm{Mn} 2 \mathrm{p}$ 特征峰的元素内层电子的结合能
差异太小, 很难推断锰的价态; 可采用 $\mathrm{Mn} 3 \mathrm{~s}$ 峰的 两个分裂峰位置的差值来评估锰的价态。样品 CMO-1 的 Mn3s 分裂峰位置差值 $\left(\Delta E_{\mathrm{b}}=5.13 \mathrm{eV}\right)$ 小 于样品 CMO-2 的 $M n 3 s$ 分裂峰位置差值 $\left(\Delta E_{\mathrm{b}}=\right.$ $5.34 \mathrm{eV})$, 表明样品 CMO-1 的表面锰平均价态高 于样品 $\mathrm{CMO}-2$ 。

\section{2 电化学性能测试}

\subsubsection{Ca-Mn-O 催化剂线性扫描伏安曲线}

为了考察样品对氧的催化性能, 对样品进行了 循环伏安测试(图 6)。首先向电解质溶液中通入氮气 进行除氧操作, 测试样品修饰电极的伏安曲线, 然 后再向溶液中通入氧气至饱和，再进行测试。从图 6 中可以看到, 电解质溶液在无氧情况下, 循环伏安 曲线没有任何特征峰出现; 通入氧气后, 在 $0.5 \mathrm{~V}$ 处 出现了明显的氧还原峰, 说明制备的两种 Ca-Mn-O 氧化物对氧还原有一定的催化活性。

\subsubsection{Ca-Mn-O 催化 ORR 的动力学性质}

图 7 是使用旋转环盘电极(RRDE)对两种材料进 行 ORR 催化性能测试的结果。图 7(a)是在转速为
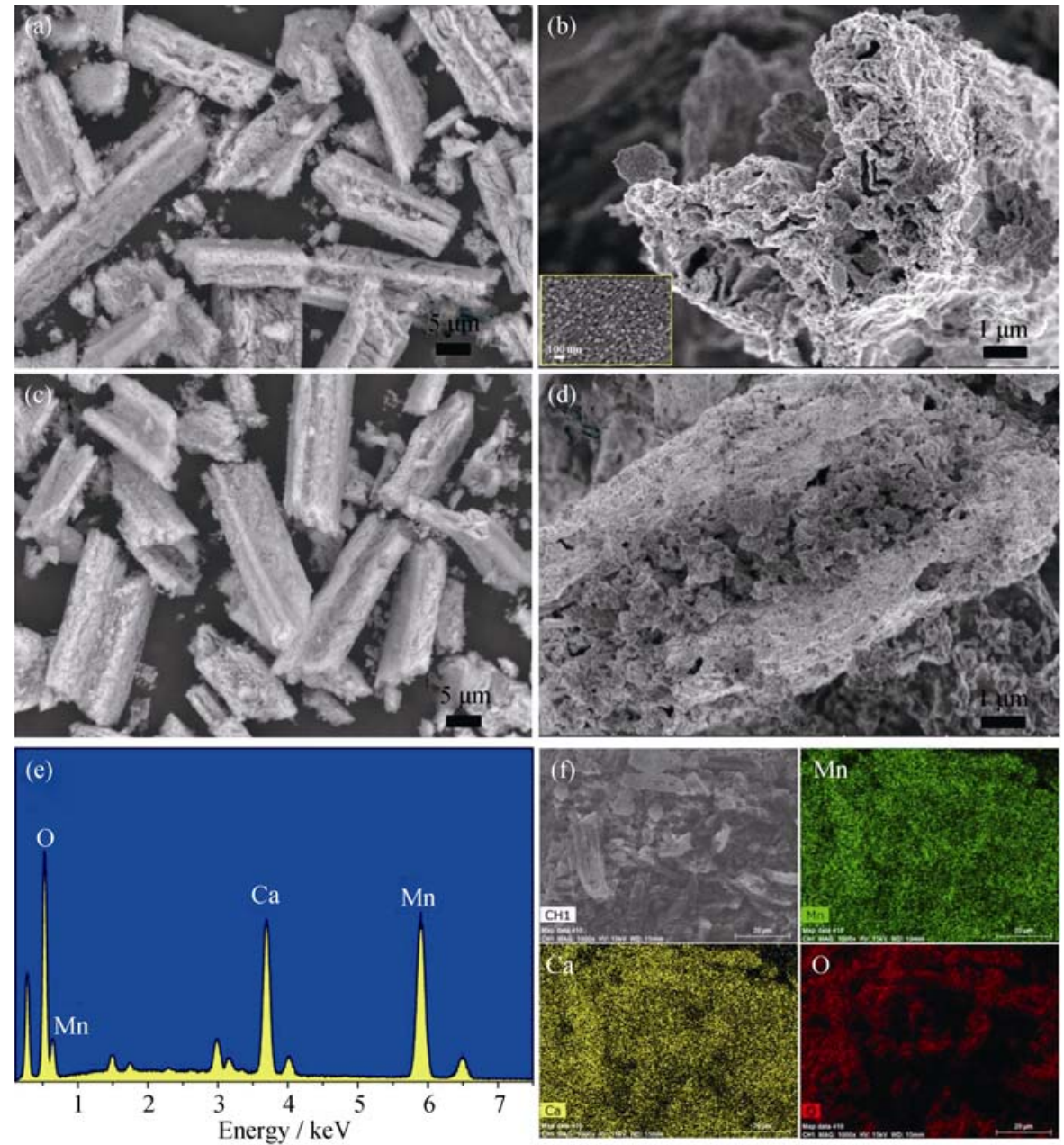

图 3 CMO-1 (a, b) 和 CMO-2(c, d) 样品的 FE-SEM 图片; CMO-1(e, f)的 EDS 分析结果

Fig. 3 FE-SEM images of CMO-1 (a, b) and CMO-2 (c, d); EDS results of CMO-1 (e, f) 

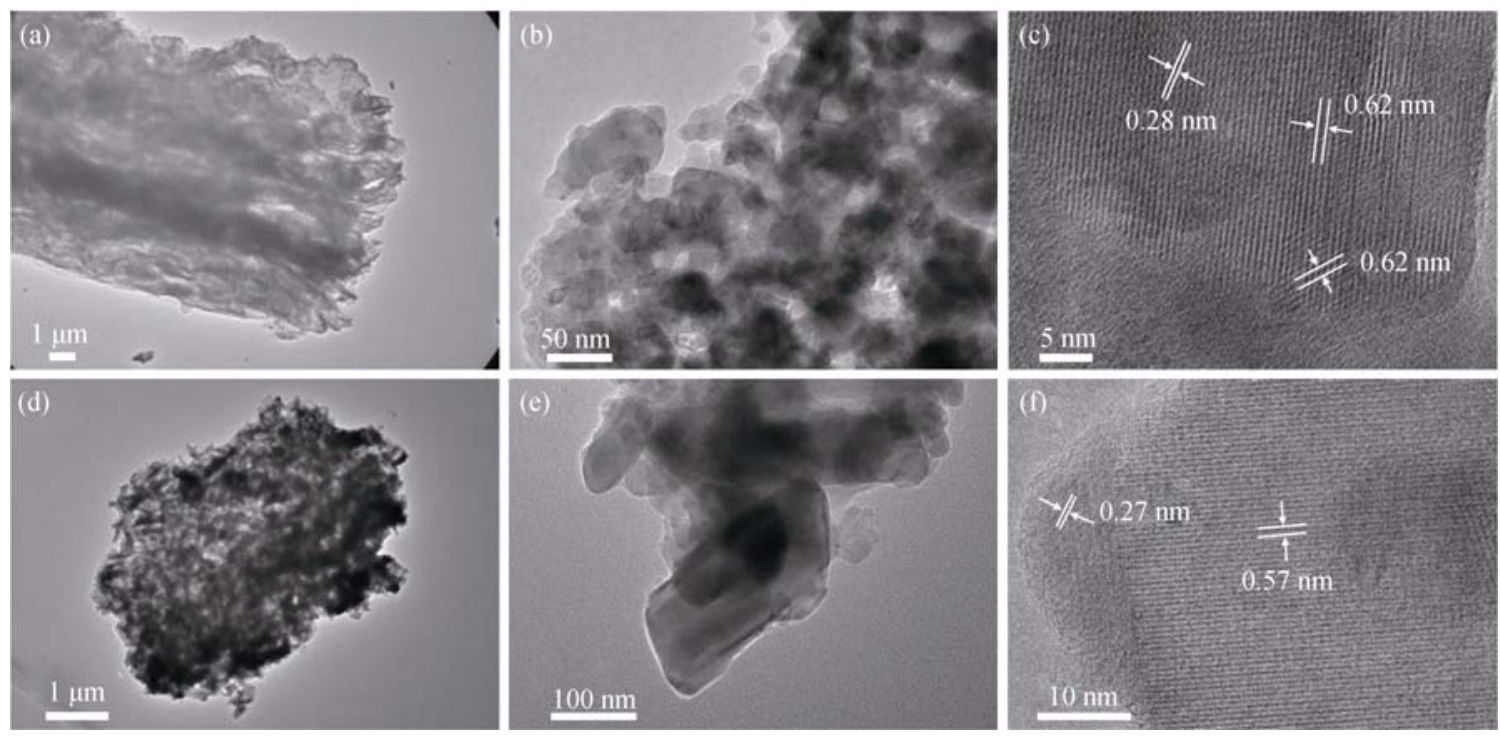

图 4 CMO-1 $(\mathrm{a} \sim \mathrm{c})$ 和 CMO-2(d f) 的 TEM 和 HRTEM 照片

Fig. 4 TEM and HRTEM images of CMO-1(a-c) and CMO-2 (d-f)
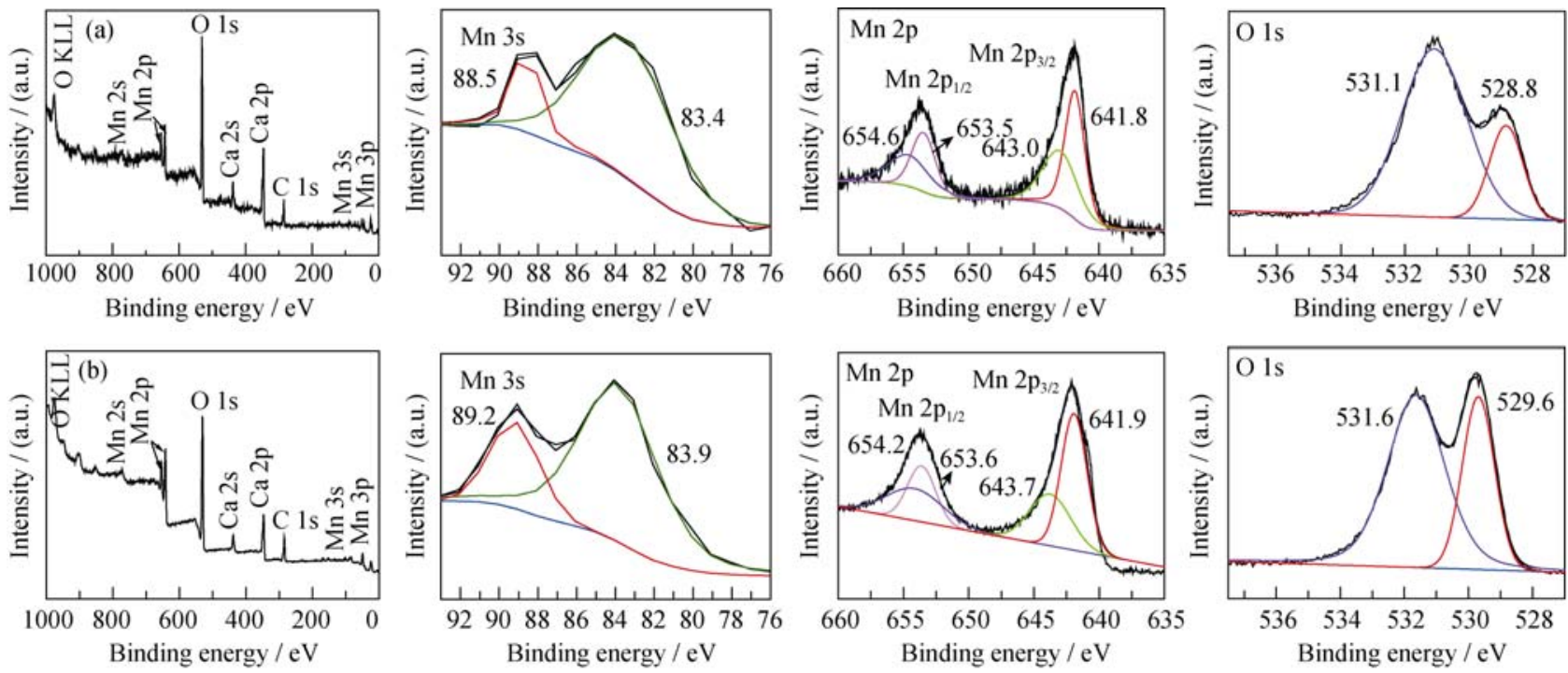

图 5 Ca-Mn-O 材料的 XPS 图谱

Fig. 5 XPS spectra of Ca-Mn-O samples (a) $\mathrm{CMO}-1$; (b) $\mathrm{CMO}-2$

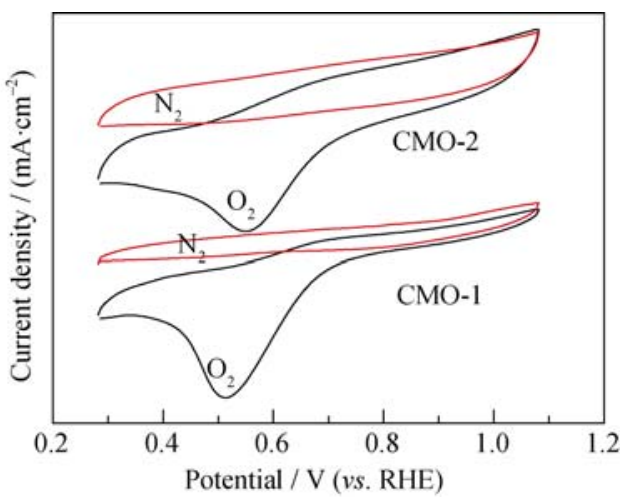

图 6 在氮气饱和 $\mathrm{KOH}$ 中 $\mathrm{Ca}-\mathrm{Mn}-\mathrm{O}$ 材料的的循环伏安曲线

Fig. $6 \mathrm{CV}$ curves of Ca-Mn-O samples in $\mathrm{N}_{2}$-saturated $0.1 \mathrm{~mol} / \mathrm{L} \mathrm{KOH}$
$1600 \mathrm{r} / \mathrm{min}$, 电位扫速为 $10 \mathrm{mV} / \mathrm{s}$ 条件下, 两种催化 剂的电化学还原反应极化曲线。图 7(b)为过氧化氢 产率和反应的电子转移数, 它是通过下面等式进行 计算得到的:

$$
\mathrm{HO}_{2}^{-}(\%)=100 \frac{2 I_{\mathrm{r}} / N}{I_{\mathrm{d}}+\left(I_{\mathrm{r}} / N\right)} \quad n=4 \frac{I_{\mathrm{d}}}{I_{\mathrm{d}}+\left(I_{\mathrm{r}} / N\right)}
$$

其中 $N$ 代表 RRDE 中的电流收集常数, $I_{\mathrm{d}}$ 和 $I_{\mathrm{r}}$ 分别 为旋转圆盘电极的盘电流密度和环电流密度。图 7(a) 表明 CMO-1 具有高的极限扩散电流密度, 与 CMO-2 相当的初始电位和半波电位; 从图 7(b)可知, 在 0.5 到 $0.7 \mathrm{~V}$ 之间, 催化剂 CMO- 1 表现出准四电子 过程和较低的过氧化氢产率, 详细的计算结果如表 1 

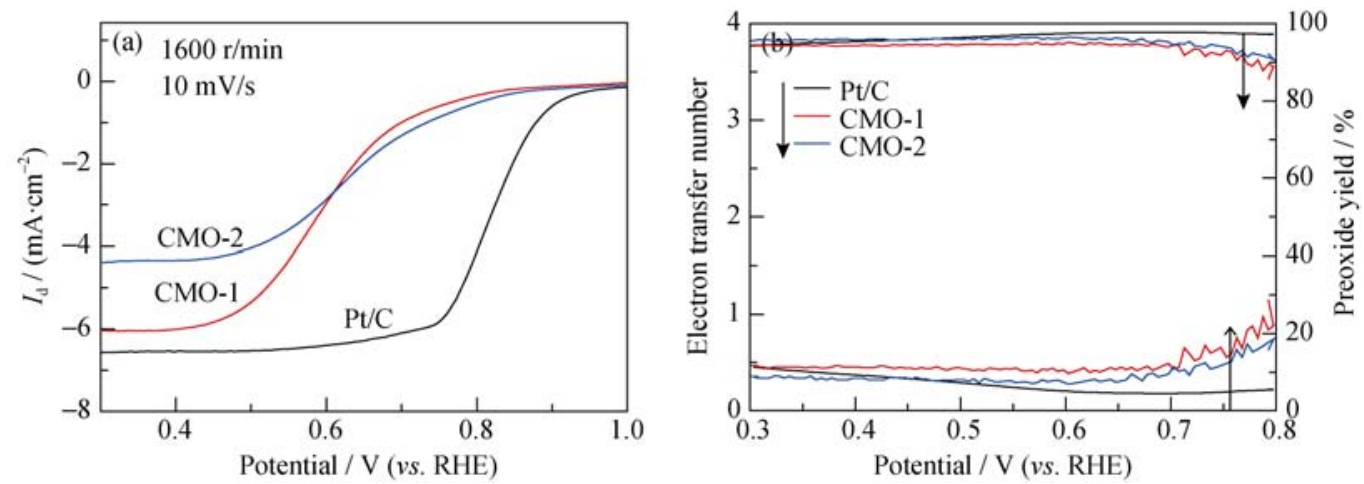

图 7 Ca-Mn-O 材料和 $\mathrm{Pt} / \mathrm{C}$ 的线性扫描伏安图(a), $\mathrm{H}_{2} \mathrm{O}_{2}$ 产率和电子转移数(b)

Fig. 7 LSVs of Ca-Mn-O samples and Pt/C (a), percentage of peroxide and electron transfer number at different potentials (b)

表 1 Ca-Mn-O 材料的电化学测试结果

Table 1 Summary of electrochemical results for the Ca-Mn-O series

\begin{tabular}{ccccccc}
\hline Material & Crystallographic structures & $E_{\text {onset }} / \mathrm{V}$ & $E_{\text {half }} / \mathrm{V}$ & $n$ & $\mathrm{HO}_{2} / \%$ & $I_{\mathrm{s}} /\left(\mathrm{mA}^{2} \cdot \mathrm{cm}^{-2}\right)(0.3 \mathrm{~V})$ \\
\hline $\mathrm{CMO}-1$ & $\mathrm{Ca}_{2} \mathrm{MnO}_{4}$ & 0.81 & 0.66 & 3.78 & $12-18$ & -6.03 \\
$\mathrm{CMO}-2$ & $\mathrm{CaMn}_{3} \mathrm{O}_{6}$ & 0.86 & 0.68 & 3.84 & $9-15$ & -4.41 \\
$\mathrm{Pt} / \mathrm{C}$ & - & 0.93 & 0.86 & 3.87 & $5-11$ & -6.38 \\
\hline
\end{tabular}

所列。CMO-1 的初始电位和半波电位分别为 $0.81 \mathrm{~V}$ 和 $0.66 \mathrm{~V}$, 电子转移数为 3.78 , 极限电流密度能够 达到-6.03 mA/ $\mathrm{cm}^{2} @ 0.3 \mathrm{~V}$, 说明相对于 $\mathrm{Pt} / \mathrm{C}$ 和 $\mathrm{CaMn}_{3} \mathrm{O}_{6}$ 而言, 催化剂 $\mathrm{Ca}_{2} \mathrm{MnO}_{4}$ 表现出准四电子过 程及与 $\mathrm{CaMn}_{3} \mathrm{O}_{6}$ 相当的电催化活性。此外, CMO-1 与 $\mathrm{CMO}-2$ 的电催化活性的差异可能与比表面积、 $\mathrm{Mn}$ 的平均表面价态和晶体结构有关 ${ }^{[15,17]}$ 。其中 CMO-1 表现出较大的极限扩散电流密度, 可能是 由于该复合物的多孔网状结构和表面均匀小颗粒, 使其具有更大的比表面积和更丰富的孔结构，进 而增多了氧还原反应的电催化活性位。并且, CMO-1 的表面吸附氧与晶格氧的峰值强度比大于 CMO-2 中对应的值, 即具有更高的氧空位浓度, 而高的氧空位浓度有利于氧还原反应的进行。此
外，虽然 CMO-1 中 $\mathrm{Mn}$ 的平均表面价态高于 CMO-2, 对催化活性有利, 但 CMO-1 层状-钙钛 矿结构中堆积密集的边角共享 $\mathrm{MnO}_{6}$ 八面体结构 不利于氧的吸附; 而 CMO-2 的尖晶石结构中, 由 于存在三分之一的 $\mathrm{Ca}$ 空位，提供了开放的空间， 使氧更容易进入，有利于 $\mathrm{O}-\mathrm{O}$ 的分裂。这可能是 CMO-2 具有稍高的反应初始电位和半波电位的主 要原因。

进一步研究催化剂在不同电极转速下的线性扫 描伏安曲线, 从图 8 中看出, 在低电位时, 极限电流 密度随着转速的增大而变大, 且样品 CMO-1 比 CMO-2 具有更大的极限扩散电流密度。在 $0.66 \mathrm{~V}$ $(\mathrm{CMO}-1)$ 和 $0.73 \mathrm{~V}(\mathrm{CMO}-2)$ 以上, 氧还原反应被电 荷传递过程控制，故电流密度不会随旋转环盘电极
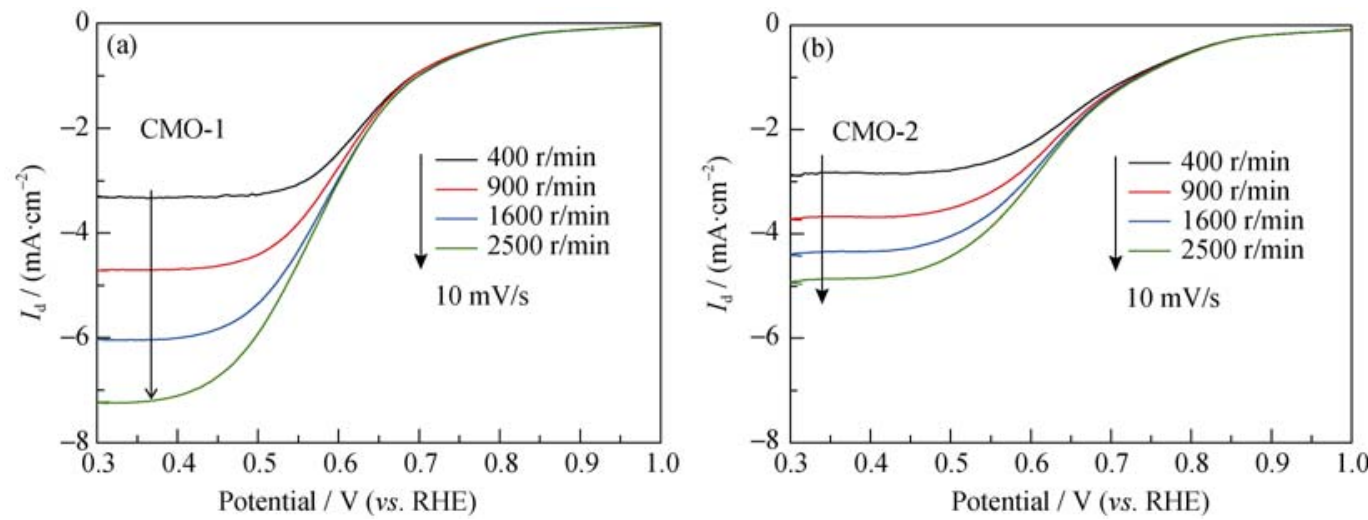

图 8 Ca-Mn-O 材料在不同转速下的线性扫描伏安图

Fig. 8 LSVs of Ca-Mn-O samples at various rotation rates 
转速而变化; 接下来 $0.66 \sim 0.56 \mathrm{~V}(\mathrm{CMO}-1)$ 和 $0.73 \sim$ $0.53 \mathrm{~V}(\mathrm{CMO}-2)$ 时, 以电荷传递-扩散混合过程为主; 之后更低电位为扩散过程。

\subsection{3 催化剂稳定性}

采用计时电流法进一步对催化剂的性能进行研 究。如图 9 所示, 在固定电位为 $0.68 \mathrm{~V}$ 下进行了 $10000 \mathrm{~s}$ 的计时电流测试, 在前 $5 \mathrm{~min}$ 内电流密度连 续减小, 最后 CMO-1 和 CMO-2 的氧还原反应电流 保持率分别在 $95.6 \%$ 和 $95.5 \%$ 。快速的衰减是由 于没有足够的气流, 以及长时间的反应使电极表 面的催化剂剥离。而 $\mathrm{Pt} / \mathrm{C}$ 的衰减与 $\mathrm{Pt}$ 纳米颗粒的 团聚和碳基体的溶解有关 ${ }^{[12]}$ 。综合所述, 层状-钙 钛矿结构的样品 CMO-1 对氧还原反应具有较高稳 定性。

\section{3 结论}

选用廉价、资源丰富的海藻酸钙纤维为载体, 经过与锰源水热处理制备了海藻纤维/锰盐复合前 驱体, 空气敾烧处理得到内部交联多孔的 $\mathrm{Ca}_{2} \mathrm{MnO}_{4}$ 产物, 并对其进行了 ORR 电催化测试。电化学性能 测试结果显示层状-钙钛矿结构的 $\mathrm{Ca}_{2} \mathrm{MnO}_{4}$ 表现出 较高的催化活性和极限扩散电流密度, 电子转移数 与标准的 $\mathrm{Pt} / \mathrm{C}$ 性能相当。此外, $\mathrm{Ca}_{2} \mathrm{MnO}_{4}$ 与对比样 $\mathrm{CaMn}_{3} \mathrm{O}_{6}$ 催化活性的差异与比表面积、锰的表面 价态和晶体结构有关。大的比表面积和丰富的孔 结构, 增多了氧还原反应的电催化活性位; 高的 氧空位浓度有利于氧还原反应的进行; 合适的晶 体结构具有开放的空间, 有利于氧的吸附。这些因 素共同决定了 $\mathrm{Ca}-\mathrm{Mn}-\mathrm{O}$ 氧化物具有较好的 ORR 电 催化性能。

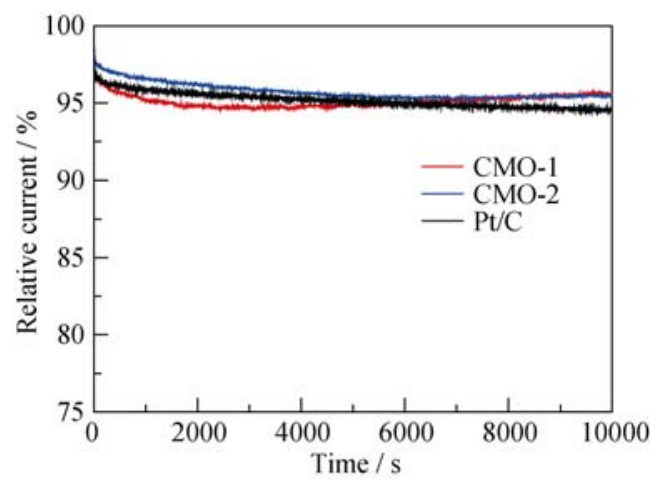

图 $9 \mathrm{Ca}-\mathrm{Mn}-\mathrm{O}$ 材料和 $\mathrm{Pt} / \mathrm{C}$ 催化剂上氧还原的计时电流 曲线

Fig. 9 Chronoamperometric curves of Ca-Mn-O samples and $\mathrm{Pt} / \mathrm{C}$

\section{参考文献:}

[1] STEELE BRIAN-C H, HEINZEL ANGELIKA. Materials for fuel-cell technologies. Nature, 2001, 414(6861): 345-352.

[2] RALPH T R, HOGARTH M P. Catalysis for low temperature fuel cells. Platinum Metals Review, 2002, 46(1): 3-14.

[3] MALAVASI LORENZO, FISHER CRAIG-A J, ISLAM M-SAIFUL. Oxide-ion and proton conducting electrolyte materials for clean energy applications: structural and mechanistic features. Chemical Society Reviews, 2010, 39(5): 4370-4387.

[4] GASTEIGER HUBET-A, KOCHA SHYAM-S, SOMPALLI BHASKAR, et al. Activity benchmarks and requirements for Pt, Pt-alloy, and non-Pt oxygen reduction catalysts for PEMFCs. Applied Catalysis B Environmental, 2005, 56(1/2): 9-35.

[5] BING YONG-HONG, LIU HAN-SAN, ZHANG LEI, et al. Nanostructured Pt-alloy electrocatalysts for PEM fuel cell oxygen reduction reaction. Cheminform, 2010, 39: 2184-2202.

[6] YANCEY DAVID-F, ZHANG LIANG, CROOKS RICHARD-M, et al. $\mathrm{Au} @ \mathrm{Pt}$ dendrimer encapsulated nanoparticles as model electrocatalysts for comparison of experiment and theory. Chemical Science, 2012, 3(4): 1033-1040.

[7] SUNTIVICH J, GASTEIGER H-A, YABUUCHI N, et al. Design principles for oxygen-reduction activity on perovskite oxide catalysts for fuel cells and metal-air batteries. Nature Chemistry, 2011, 3(7): 546-550.

[8] EL-DEAB MOHAMED-S, OHSAKA TAKEO. Manganese oxide nanoparticles electrodeposited on platinum are superior to platinum for oxygen reduction. Angewandte Chemie International Edition, 2006, 45(36): 5963-5966.

[9] ROCHE I, CHAINET E, CHATENT M, et al. Carbon-supported manganese oxide nanoparticles as electrocatalysts for the oxygen reduction reaction (ORR) in alkaline medium: physical characterizations and ORR mechanism. Journal of Physical Chemistry C, 2007, 111(3): 1434-1443.

[10] ROCHE I, CHATENET E, CHATENET M, et al. Durability of carbon-supported manganese oxide nanoparticles for the oxygen reduction reaction (ORR) in alkaline medium. Journal of Applied Electrochemistry, 2008, 38(9): 1195-1201.

[11] GE XIAOMING, LIU YAYUAN, THOMAS GOH F W, et al. Dual-phase spinel $\mathrm{MnCo}_{2} \mathrm{O}_{4}$ and spinel $\mathrm{MnCo}_{2} \mathrm{O}_{4} /$ nanocarbon hybrids for electrocatalytic oxygen reduction and evolution. $A C S A p$ plied Materials \& Interfaces, 2014, 6(15): 12684-12691.

[12] LIANG YONG-YE, WANG HAI-LIANG, ZHOU JI-GANG, et al. Covalent hybrid of spinel manganese-cobalt oxide and graphene as advanced oxygen reduction electrocatalysts. Journal of the American Chemical Society, 2012, 134(7): 3517-3523.

[13] YANG HONG-CHAO, HU FENG, ZHANG YE-JUN, et al. Con- 
trolled synthesis of porous spinel cobalt manganese oxides as efficient oxygen reduction reaction electrocatalysts. Nano Research, 2016, 9(1): 201-213.

[14] CHENG FANG-YI, SU YI, LIANG JING, et al. $\mathrm{MnO}_{2}$-based nanostructures as catalysts for electrochemical oxygen reduction in alkaline media. Chemistry of Materials, 2010, 22(3): 898-905.

[15] LIMA FABIO H B, CALEGARO MARCELO L, TICIANELLI EDSON A. Investigations of the catalytic properties of manganese oxides for the oxygen reduction reaction in alkaline media. Biological Journal of the Linnean Society, 1996, 57(1): 1-11.

[16] HU YU-XIANG, HAN XIAO-PENG, ZHAO QING, et al. Porous perovskite calcium-manganese oxide microspheres as an efficient catalyst for rechargeable sodium-oxygen batteries. Journal of Materials Chemistry A, 2015, 3: 3320-3324.

[17] HAN XIAO-PENG, ZHANG TIAN-RAN, DU JING, et al. Porous calcium-manganese oxide microspheres for electrocatalytic oxygen reduction with high activity. Chemical Science, 2012, 4(1):
$368-376$.

[18] PENG HONG-LIANG, LIU FANG-FANG, LIU XIAO-JUN, et al. Effect of transition metals on the structure and performance of the doped carbon catalysts derived from polyaniline and melamine for ORR application. ACS Catalysis, 2014, 4(10): 3797-3805.

[19] YANG XIAN-FENG, FU JUN-XIANG, JIN CHONG-JUN, et al. Formation mechanism of $\mathrm{CaTiO}_{3}$ hollow crystals with different microstructures. Journal of the American Chemical Society, 2010, 132(40): 14279-14287.

[20] HAN XIAO-PENG, CHENG FANG-YI, ZHANG TIAN-RAN, et al. Hydrogenated uniform Pt clusters supported on porous $\mathrm{CaMnO}_{3}$ as a bifunctional electrocatalyst for enhanced oxygen reduction and evolution. Advanced Materials, 2014, 26(13): 2047-2051.

[21] HU JIE, WANG LI-NA, SHI LI-NA, et al. Preparation of $\mathrm{La}_{1-\mathrm{x}} \mathrm{Ca}_{\mathrm{x}} \mathrm{MnO}_{3}$ perovskite-graphene composites as oxygen reduction reaction electrocatalyst in alkaline medium. Journal of Power Sources, 2014, 269(269): 144-151. 